\title{
Homozygosity for the HLA-DRB1 Allele Selects for Extraarticular Manifestations in Rheumatoid Arthritis
}

\author{
Cornelia M. Weyand, Congping Xie, and Jörg J. Goronzy \\ Department of Medicine, Division of Rheumatology, Mayo Clinic and Foundation, Rochester, Minnesota 55905
}

\begin{abstract}
Seropositive rheumatoid arthritis is genetically linked to a group of HLA-DRB1 alleles sharing a sequence motif within the third hypervariable region. Controversy exists over the role of the distinct allelic variants in affecting not only the risk to develop disease, but also in modifying the expression of the disease. We have stratified 81 patients according to their patterns of disease manifestations and identified the HLA-DRB1 alleles by polymerase chain reaction amplification and subsequent oligonucleotide hybridization. To identify precisely the allelic combinations at the HLA-DRB1 locus, homozygosity was confirmed by locus-specific cDNA amplification and subsequent sequencing. Our study demonstrated a high correlation of allelic combinations of disease-associated HLA-DRB1 alleles with the clinical manifestations. Characteristic genotypes were identified for patients who had progressed toward nodular disease and patients who had developed major organ involvement. Rheumatoid nodules were highly associated with a heterozygosity for two disease associated HLA-DRB1 alleles. Homozygosity for the HLA-DRB1*0401 allele was a characteristic finding for RA patients with major organ involvement. Our data suggest a role of the disease-associated sequence motif in determining severity of the disease. The finding of a codominant function of HLA-DRB1 alleles suggests that the biological function of HLA-DR molecules in thymic selection might be important in the pathogenesis of RA. (J. Clin. Invest. 1992. 89:2033-2039.) Key words: rheumatoid arthritis • HLA-DRB1 alleles $\bullet$ rheumatoid nodules $\bullet$ rheumatoid vasculitis $\bullet$ prognostic markers
\end{abstract}

\section{Introduction}

Seropositive rheumatoid arthritis is the most common autoimmune disease afflicting some 4-6 million Americans. Conservative estimates predict $100,000-200,000$ new cases of RA each year (1). The syndrome is characterized by a chronic destructive inflammatory process, mainly expressed in the joints and often resulting in permanent loss of joint function. The sys-

Address reprint requests and correspondence to Cornelia $M$. Weyand, M. D., Ph. D., 401 Guggenheim, Mayo Clinic, 200 First Street SW, Rochester, MN 55905

Received for publication 10 December 1991 and in revised form 11 February 1992

The Journal of Clinical Investigation, Inc.

Volume 89, June 1992, 2033-2039 temic nature of the disease is demonstrated by extraarticular manifestations which may manifest as vasculitis, cardiopulmonary involvement, hematologic abnormalities, and neurovascular disease (2). In the subgroup of patients with significant extraarticular disease, RA is a serious and even life-threatening illness warranting intensive therapeutic measures. Therapeutic management of active RA is limited to pain control, antiinflammatory agents, and nonselective immunosuppression. No curative therapeutic intervention is available. Currently more aggressive therapy of the syndrome is hampered by the lack of predictability of the course of the disease in individual patients. No useful prognostic markers have been established which would allow us to identify patients at risk to develop the severe crippling or life-threatening form of the disease before major organ damage has occurred.

Although the pathogenesis of this inflammatory autoimmune disorder has not been elucidated, RA is now understood as an entity with a strong genetic component. A set of alleles at the B1 locus of the HLA-DR region, HLA-DRB $1{ }^{*} 0401$, * $^{*} 0404$, ${ }^{*} 0405,{ }^{*} 0408,{ }^{*} 0101$, and ${ }^{*} 1402$ is highly associated with the disease (3-7). All disease-associated haplotypes share a specific polymorphism encoded by the third hypervariable region of the HLA-DRB 1 gene suggesting that the shared sequence motif is involved in the pathogenesis of RA (8-10). Whether the disease-associated alleles contribute directly or indirectly to the disease process is unclear, but all of them have been defined as susceptibility markers.

We have hypothesized that the gene products of the HLADRB1 locus have a pathogenetic role over and above their dominant effect in conferring susceptibility to RA. In particular, we wanted to study whether heterozygosity or homozygosity of the disease-associated B1 alleles might modify the expression of the disease in individual patients. To investigate the relationship between the allelic products encoded by both $B 1$ genes and the severity of the disease, we have identified allelic combinations of HLA-DRB1 genes in three subsets of RA patients classified based upon their disease manifestations. Here we report that the function of the HLA-DRB1 locus in RA extends beyond the role of a susceptibility marker. Characteristic genotypes were identified for patients with distinct manifestations of RA. Among the disease-associated alleles, the $B 1 * 0401$ variant played a particular role and selected for disease progression. Combinations of DRB $1{ }^{*} 0401$ with B $1 * 0404$ / 8 or ${ }^{*} 0101$ was the typical genotype for patients with nodular RA. Homozygosity for the ${ }^{*} 0401$ gene at the DRB1 locus was the characteristic finding for patients with involvement of major organ systems. The finding of a codominant function of HLA-DRB1 alleles questions the model that the disease-associated polymorphism is pathogenetic by selecting and presenting an arthritogenic antigen but rather suggests that the disease- 
associated sequence polymorphism is functional in modifying the patients' $\mathrm{T}$ cell receptor $(\mathrm{TCR})^{1}$ repertoire.

\section{Methods}

Patients. We examined a total of 81 patients with RA in this study. All patients were diagnosed and treated at the Division of Rheumatology, Mayo Clinic. After obtaining informed consent, patients were referred for drawing of a blood sample. Patients selected for the study fulfilled the diagnostic criteria of the American College of Rheumatology for RA (11), carried a positive rheumatoid factor, and had erosive disease as documented by hand $x$ rays. The characteristics of the patients are described in Table I. They were divided into three groups based on the criteria described below. Group 1 comprised 27 patients with RA but no rheumatoid nodules or any other extraarticular manifestations. Group 2 comprised 36 patients. All of them presented with rheumatoid nodules but had no other extraarticular disease. Group 3 comprised 18 patients with unequivocal extraarticular manifestations of RA in addition to nodules. The group included patients with rheumatoid vasculitis, with rheumatoid lung disease presenting as interstitial lung fibrosis, and with Felty's syndrome. Rheumatoid vasculitis was diagnosed by histomorphology or angiogram. Nonhealing ulcers were classified as rheumatoid ulcers if the upper extremities were involved or lower extremity ulcerations occurred in the absence of arteriosclerotic disease. Periungual nailfold infarcts in the absence of any other extraarticular manifestation of RA or leg ulcers in the presence of arteriosclerosis were not considered sufficient to document the diagnosis of rheumatoid vasculitis (12). The control group comprised 47 healthy individuals with no personal or family history of RA. All patients and all control individuals were Caucasian.

DNA analysis. Genomic DNA was isolated from peripheral blood mononuclear cells as described $(13,14)$. DRB1 alleles were amplified by a polymerase chain reaction (PCR) with the following primers: HLA-DR1, TTGTGGCAGCTTAAGTTTGAA (codons 8-14); HLADR2, TTCTTGCAGCAGGATAAGTATG (codons 7-13); HLADR3, 5, w6, TTCTTGGAGTACTCTACGTCTGAG (codons 7-14); HLA-DR4, GTTTCTTGGAGCAGGTTAAAC (codons 6-12); HLA-DR7, TTCCTGTGGCAGGGTAAGTATA (codons 7-13); HLA-DRw8, TTCTTGGAGTACTCTACGGGTG (codons 7-13); HLA-DR9, TTCTGGAAGCAGGATAAGTTTG (codons 7-13); HLA-DRw10, GAGGAGGTTAAGTTTGAGTGTC/ (codons 9-15); and GCCGCTGCACTGTGAAGCTCTC (DRB1 codons 93-87, nonpolymorphic). Amplification was carried out for 30 cycles consisting of 2 min denaturation at $94^{\circ} \mathrm{C}, 2 \mathrm{~min}$ annealing at $55^{\circ} \mathrm{C}$, and $2 \mathrm{~min}$ extension at $72^{\circ} \mathrm{C}$. PCR products were subsequently separated by gel electrophoresis and stained with ethidium bromide. Each PCR reaction included a negative and positive control. Genomic DNA extracted from homozygous typing lines representing the various DRB1 alleles served as individual controls for the allele-specific primer sets.

Oligonucleotide hybridization of amplified DNA. PCR products were analyzed for efficiency and specificity of the reaction by electrophoresis and adjusted to comparable concentrations. Subsequently, the amplified fragments were blotted and hybridized to allele specific oligonucleotides as described $(13,14)$. The following probes were used to detect polymorphism of the DR4 alleles and to define the HLA-DR3, 5, w13, and w 14 alleles: DRB $1 * 0401$, CGCGGCCCGCTTCTGCTCC (codons 69-74); B1*0402, B1*1301/2, CGCGGCCCGCTCGTCTTCC (codons 69-74); B1*0404/8, *0405, *1402, CACCGCGGCCCGCCTCTGC (codons 70-75); B1*0403, CACCTCGGCCCGCCTCTGC (codons 70-75); CTACGGGGTTGGTGAGAGC (codons 83-88, with glycine at position 86); HLA-DR3, AAGCGGGGCCGGGTGGACAAC (codons 71-77), and HLA-DR5, TATAACCAAGAGGAGTACGTG (codons 31-37). Probes were labeled

1. Abbreviations used in this paper: TCR, T cell receptor; PCR, polymerase chain reaction.
Table I. Characteristics of the Study Populations

\begin{tabular}{lccc}
\hline & \multicolumn{3}{c}{ Rheumatoid arthritis patients } \\
\cline { 2 - 4 } & Group 1 & Group 2 & Group 3 \\
\hline $\begin{array}{l}\text { Nodules } \\
\text { Additional extraarticular }\end{array}$ & - & + & + \\
$\quad$ manifestations & - & - & + \\
$\begin{array}{l}\text { Number of patients } \\
\text { Age }(y r)\end{array}$ & 27 & 36 & 18 \\
$\quad \begin{array}{l}\text { Median } \\
\text { Range }\end{array}$ & 58 & 61 & 62.5 \\
$\begin{array}{l}\text { Sex (m/f) } \\
\text { Disease duration }(y r)\end{array} \quad 39-78$ & $29-81$ & $39-84$ \\
$\quad$ Median & $3 / 24$ & $17 / 19^{*}$ & $8 / 10^{\ddagger}$ \\
$\quad$ Range & 11 & 13 & 14 \\
Age at disease onset $(y r)$ & $3-43$ & $3-32$ & $3-33$ \\
$\quad$ Median & & & \\
Range & 46 & 49.5 & 47.5 \\
& $15-61$ & $18-69$ & $17-72$
\end{tabular}

${ }^{*} P=0.0023 ;{ }^{\ddagger} P=0.0108$.

with digoxigenin-UTP, blots were developed with digoxigenin-specific alkaline phosphatase-labeled antibodies as described by the manufacturer (Genius; Boehringer Mannheim Corp., Indianapolis, IN). Each experiment included appropriate hybridization controls of DNA from homozygous typing lines defined by The Tenth International Histocompatibility Workshop (15).

Sequence analysis of the HLA-DRBI locus. Total RNA was extracted from PBMC by guanidium thiocyanate phenol chloroform extraction using RNAzol (Cinna/Biotex, Friendswood, TX). cDNA was synthesized and amplified using a primer set inclusive for all B1 encoded products, ATGGTGTGTCTGAAGCTCCC (codons -29 to -23) and TTCAGACCGTGCTCTCCATTC (codons 193-186). A T7 phage promotor was attached to the primer corresponding to the leading sequence position -29 to -23 . After denaturation $3 \mu$ of the PCRgenerated templates were mixed with $17 \mu \mathrm{l}$ of a RNA transcription mixture (40 mM Tris HCL, pH 7.5, $6 \mathrm{mM} \mathrm{MgCl}, 2 \mathrm{mM}$ spermidine, 10 $\mathrm{mM} \mathrm{NaCl}, 0.5 \mathrm{mM}$ of the four ribonucleoside triphosphates, $10 \mathrm{mM}$ DTT, $32 \mathrm{U}$ RNasin, and $10 \mathrm{U}$ T7 RNA polymerase). After $3 \mathrm{~h}$ of incubation at $37^{\circ} \mathrm{C}, 2 \mu \mathrm{l}$ of the transcript were directly sequenced by transcriptase-mediated dideoxysequencing $(16,17)$. A ${ }^{32}$ P-endlabeled internal primer (codons 93-87) was used as a reverse transcriptase primer to increase the selectivity of the sequence reaction. Unambiguous sequences were obtained for homozygous patients.

Statistical analysis. Demographic features of the three study populations were compared by Wilcoxon's signed rank test. The significance of association of HLA-DRB1 alleles with the clinical subsets of RA was assessed using a chi-square test or a Fisher's exact probability test, if appropriate.

\section{Results}

Identification of both $H L A-D R B 1$ alleles. To examine the allelic polymorphism of the HLA-DRB1 locus at the molecular level, we applied PCR in combination with oligonucleotide hybridization. To confirm homozygosity in HLA-DR4 ${ }^{+}$patients, the B1 gene was amplified with allele-nonspecific primers and sequenced. Initially, the genomic DNA of each patient was amplified with a set of primers selective for the allelic polymorphism of the first hypervariable region and a nonpolymorphic primer of the second exon of the B1 locus. As demonstrated in Fig. $1 a$ this reaction resulted in the amplifica- 

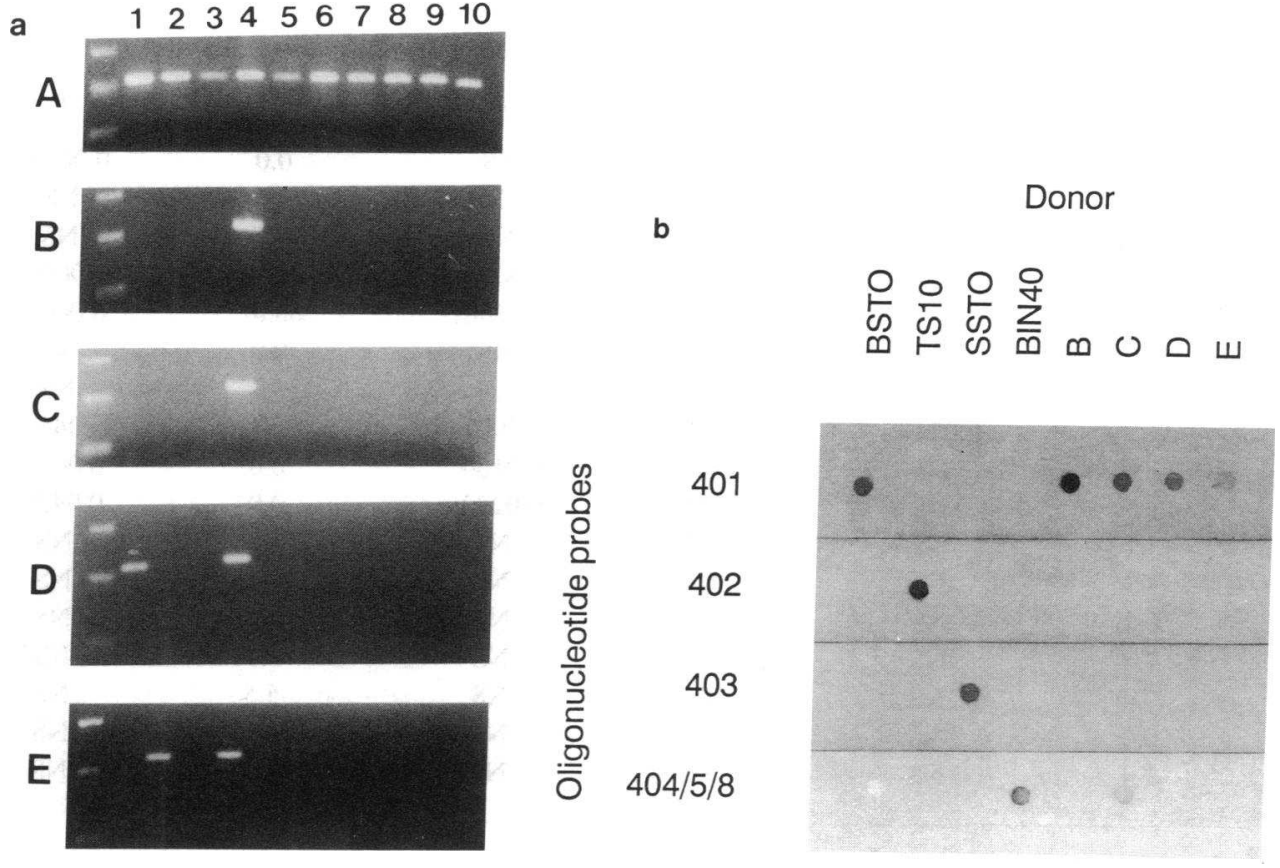

Figure 1. HLA-DRB1 genotyping. DNA from homozygous typing lines representing the HLA-DR haplotypes 1 through w10 $(A)$ was amplified with DRB1 allele-specific primer sets (a). The lane number indicates the HLA-DR haplotype amplified. Amplification products from four different RA patients are shown in rows $B-E$. Only one product was amplified for patients $B$ and $C$, whereas the products of two different alleles were identified for patients D and E. Products amplified with the HLA-DR4 specific primers were blotted and hybridized with oligonucleotides specific for the distinct HLA-DR4 subtypes (b). The homozygous typing lines BST0 (HLA-DRB1*0401), TS10 (HLA-DRB1*0402), SSTO (HLA-DRB1*0403), and Bin 40 (HLA-DRBI*0404) were used as controls. tion of an $\sim 260$-bp fragment and allowed the unambiguous assignment of $B 1$ alleles in RA patients combining the DR4 haplotype with a non-DR4 haplotype. The HLA-DR4 haplotype includes a heterogeneous group of DRB1 alleles not all of which are associated with RA. To dissect the complexity of the DRB1 alleles (18), HLA-DR4 ${ }^{+}$individuals were further studied for allelic variants by blotting and hybridization with oligonucleotide probes specific for the sequence polymorphism at amino acid positions $67,70,71,74$, and 86 . Fig. $1 b$ shows blots from representative patients. Oligonucleotide reaction patterns allowed the identification of individuals who appeared homozygous by PCR amplification for the DRB1*04 sequence polymorphism but carried two distinct HLA-DR4 subtypes, usually the *0401 and *0404 alleles (Fig. 1, patient C). We did not attempt to determine the polymorphism at position 57 which is more relevant for an Asian population. Our study therefore could not distinguish between the rare alleles HLADRB 1*0405 and *0408 (Fig. 1 b). In case allele-specific PCR amplification followed by oligonucleotide probing detected only one single product in a patient, e.g., HLA-DRB $1 * 0401$ in patient B (Fig. 1), we confirmed the homozygous state for the HLA-DRB 1 gene by sequence analysis to exclude the possibilities that either a variant HLA-DRB1 allele had been overlooked in the initial DNA amplification $(13,19)$ or that a polymorphism within the HLA-DR4 alleles was not detected in the subsequent hybridization. In addition, the HLA-DRB1 locus of four patients was sequenced whose initial typing results were equivocal. To include all possible HLA-DRB1 alleles in the analysis, cDNA was amplified by using an oligonucleotide corresponding to the leader sequence of the HLA-DRB1 locus and a second primer encoding for a polymorphism shared by all HLA-DRB1 alleles, but distinct for the HLA-DRB4 locus. Thus, in HLA-DR4 ${ }^{+}$individuals homozygous at the HLADRB1 locus only one amplified product is expected. Amplified cDNA was transcribed and subsequently directly sequenced by reverse transcriptase-mediated dideoxysequencing $(16,17)$.
The procedure allowed us to determine whether a patient transcribes a single or two different alleles encoded by the HLADRB1 gene. By combining PCR amplification, oligonucleotide hybridization, and sequence analysis, we were able to unequivocally assign genotypes for both $\mathrm{B} 1$ alleles in all patients studied. The sequence analysis required the reassignment of HLADRB1 typing in one patient. One additional RA patient with extraarticular manifestations, who could not be typed by serological typing or genomic amplification, we found to be heterozygous and most closely resembling the HLA-DRB1*1401 and B $1 * 1402$ alleles with two mutations (data not shown).

Immunogenetic profile of $R A$ patients with erosive nonnodular disease. Erosive nonnodular RA was highly associated with the sequence polymorphism shared by the previously implicated HLA-DR alleles (Table II). $92.5 \%$ of the patients expressed at least one of the disease-associated HLA-DRB1 alleles. The $B 1^{*} 0401$ allele was the most frequent subtype present in that patient population and was identified in $51.9 \%(P$ $=0.0016)$. Six patients were typed as $\mathrm{B} 1^{*} 0404^{+}, \mathrm{B} 1^{*} 0405^{+}$, or $B 1^{*} 0408^{+}(P=0.0241)$. In four of the seven HLA-DR4 ${ }^{-}$RA patients, the $B 1^{*} 0101$ allele, and in one, the $\mathrm{B} 1 * 1402$ allele, were detected. The two patients who did not express any disease-linked haplotype were typed as DR2/DR3 and DR5/ DR7. Two distinct HLA-DRB1 alleles were found for each of the 27 patients with nonnodular disease. None of the patients was homozygous for a disease-linked B1 allele. Three patients (11.1\%) carried a combination of two distinct RA-associated B1 genes, HLA-DRB1*0101/0401 in one patient and HLADRB1*0101/0404 in two patients (Table III). None of these combinations was more frequent than expected. In patients combining a disease-relevant $\mathrm{B} 1$ gene with a nonassociated $\mathrm{B} 1$ gene, HLA-DR3 was frequently found. Based upon the representation of the DR3 phenotype in the normal population, the DR3/DR4 heterozygosity was expected in $\sim 10 \%$ of HLADR4 ${ }^{+}$probands, but was seen in $30 \%$ of nonnodular HLADR4 ${ }^{+}$patients. 
Table II. Frequency of HLA-DR Haplotypes in the Study Populations

\begin{tabular}{|c|c|c|c|c|c|c|c|}
\hline & Normal controls & Group 1 & & Group 2 & & Group 3 & \\
\hline & $\%$ & $\%$ & $P$ & $\%$ & $P$ & $\%$ & $P$ \\
\hline DR1 & 27.6 & 25.9 & NS & 27.7 & NS & 0.0 & 0.0086 \\
\hline DR2 & 31.9 & 11.1 & 0.0447 & 11.1 & 0.0254 & 11.0 & NS \\
\hline DR3 & 21.2 & 25.9 & NS & 8.3 & NS & 5.5 & NS \\
\hline DR4 & 25.5 & 77.8 & 0.00001 & 100.0 & 0.00001 & 94.4 & 0.00001 \\
\hline 401 & 17.0 & 51.9 & 0.0016 & 83.3 & 0.00001 & 88.8 & 0.00001 \\
\hline 402 & 2.1 & 0.0 & NS & 0.0 & NS & 0.0 & NS \\
\hline 403 & 2.1 & 7.4 & NS & 0.0 & NS & 0.0 & NS \\
\hline $404 / 5 / 8$ & 4.3 & 22.2 & 0.0241 & 38.8 & 0.0001 & 22.3 & 0.0451 \\
\hline DR5 & 29.8 & 11.1 & NS & 8.3 & 0.0164 & 0.0 & 0.0056 \\
\hline DR7 & 19.1 & 18.5 & NS & 2.8 & 0.0223 & 0.0 & 0.0426 \\
\hline DRw8 & 10.6 & 3.7 & NS & 8.3 & NS & 0.0 & NS \\
\hline DR9 & 4.3 & 3.7 & NS & 0.0 & NS & 0.0 & NS \\
\hline DRw10 & 2.1 & 0.0 & NS & 0.0 & NS & 0.0 & NS \\
\hline DRw13 & 17.0 & 7.4 & NS & 5.6 & NS & 5.5 & NS \\
\hline DRw14 & 4.3 & 7.4 & NS & 0.0 & NS & 5.5 & NS \\
\hline 1401 & 2.1 & 0.0 & NS & 0.0 & NS & 5.5 & NS \\
\hline 1402 & 2.1 & 7.4 & NS & 0.0 & NS & 5.5 & NS \\
\hline
\end{tabular}

$P=$ Comparison of haplotype frequencies in patient group versus normal controls.

Immunogenetic profile of $R A$ patients with nodular disease. All patients with nodular disease were positive for the DR4 phenotype (Table II). By far the most frequent disease-linked B1 gene found in nodular patients was the B1*0401 allele which was expressed by $83.3 \%(P \leq 0.00001)$ of all individuals. Alleles that carried the ${ }^{*} 0404 / 5 / 8$ associated HVR3 sequence were identified in $38.8 \%$ of all patients and thus were strongly enriched in the patient group as well $(P=0.0001)$. The group did not include any patients expressing the $\mathrm{B} 1{ }^{*} 1402$ allele. The $\mathrm{B} 1{ }^{*} 0101$ allele was present in $27.7 \%$ individuals and thus not decreased. However, in all cases, it was combined with a disease-associated subtype of HLA-DR4. Compared to the patients with erosive nonnodular RA, the number of HLA-DR4patients, in particular patients solely expressing HLA-DR 1 as a disease-associated allele, was significantly decreased (Table III) $(P=0.0295)$. PCR analysis of genomic DNA revealed a single product in 10 of 36 patients amplified by the primer set specific for the DR4 haplotype suggesting homozygosity for that phenotype. For eight of these patients, the oligonucleotide hybridization patterns demonstrated a $B 1^{*} 0401 /{ }^{*} 0404$ or $\mathrm{B} 1{ }^{*} 0401 /$ *0408 heterozygous genotype (Table IV). Sequence analysis confirmed that one of the patients was homozygous for the B1*0401 allele, a second patient was homozygous for the B $1 * 0404$ allele. Another set of 10 nodular patients was found to be homozygous for the disease-linked sequence motif (Table IV). In these patients, the combination of a DR4 variant with the $B 1^{*} 0101$ gene was characteristic. Non-disease-associated $B 1$ alleles as the second haplotype were seen in $41.7 \%$ of patients. The distribution of nonassociated alleles was not different from that in the control group. The chief finding for the patient subset with nodular disease, the high frequency of allele combinations including two distinct disease-linked B1 genes was highly significant $(P=0.0003)$ when compared to the patients with nonnodular disease (Table III). The combination of two distinct DR4B1 variants $(P=0.0078)$ as well as the combi- nation of the $B 1^{*} 0101$ gene with $B 1 * 0401(P=0.0385)$ or $\mathrm{B} 1{ }^{*} 0404$ but not the homozygosity of a disease-associated allele was characteristic for patients with nodules and no major organ involvement.

Immunogenetic profile of patients with extraarticular disease. The patient group with extraarticular disease included individuals with a wide spectrum of clinical manifestations all

Table III. Combinations of Disease-associated HLA-DRBI Alleles in Rheumatoid Arthritis Patients Presenting with Distinct Patterns of Disease Manifestations

\begin{tabular}{lccccc}
\hline & $\begin{array}{c}\text { Nonnodular } \\
\text { disease }\end{array}$ & $\begin{array}{c}\text { Nodular } \\
\text { disease }\end{array}$ & $P^{*}$ & $\begin{array}{c}\text { Extraarticular } \\
\text { disease }\end{array}$ & $P^{\ddagger}$ \\
\hline No. of patients & 27 & 36 & & 18 & \\
Genotype & & & & & \\
B1 alleles & & & & & \\
$\quad 0401 / 0401$ & 0 & 1 & NS & 9 & 0.0001 \\
$0404 / 0404$ & 0 & 1 & NS & 1 & NS \\
$0401 / 0404(5,8)$ & 0 & 8 & 0.0078 & 3 & NS \\
$0401 / 0101$ & 1 & 8 & 0.0385 & 0 & 0.0291 \\
$0404 / 0101$ & 2 & 2 & NS & 0 & NS \\
$0401 / x^{8}$ & 13 & 13 & NS & 4 & NS \\
$0404 / x$ & 4 & 3 & NS & 0 & NS \\
$0101 / x$ & 4 & 0 & 0.0295 & 0 & NS \\
$1402(5) / x$ & 1 & 0 & NS & 1 & NS \\
$\quad$ x/x & 2 & 0 & NS & 0 & NS \\
Patients with & & & & & \\
$\quad$ two disease- & & & & & \\
$\quad$ associated & & & & & \\
$\quad$ B1 alleles & 3 & 20 & 0.0003 & 13 & NS \\
\hline
\end{tabular}

* Nodular patients versus nonnodular patients. ${ }^{\ddagger}$ Patients with major organ involvement versus nodular patients. ${ }^{8} \mathrm{x}=$ HLA-DRB1 alleles not associated with RA. 
Table IV. Allelic Combinations at the HLA-DRBI Locus in Patients with Nodular Rheumatoid Arthritis

\begin{tabular}{|c|c|c|c|c|c|}
\hline & \multicolumn{4}{|c|}{ Disease-associated B1 allel } & \multirow{2}{*}{$\begin{array}{l}\text { Nonassociated } \\
\text { B1 allele }\end{array}$} \\
\hline & 401 & $404 / 5 / 8$ & 101 & 1402 & \\
\hline $2-1$ & $+/+$ & - & - & - & - \\
\hline $2-2$ & - & $+/+$ & - & - & - \\
\hline $2-3$ & + & + & - & - & - \\
\hline $2-4$ & + & + & - & - & - \\
\hline $2-5$ & + & + & - & - & - \\
\hline $2-6$ & + & + & - & - & - \\
\hline $2-7$ & + & + & - & - & - \\
\hline $2-8$ & + & + & - & - & - \\
\hline $2-9$ & + & + & - & - & - \\
\hline $2-10$ & + & + & - & - & - \\
\hline $2-11$ & + & - & + & - & - \\
\hline $2-12$ & + & - & + & - & - \\
\hline $2-13$ & + & - & + & - & - \\
\hline $2-14$ & + & - & + & - & - \\
\hline $2-15$ & + & - & + & - & - \\
\hline $2-16$ & + & - & + & - & - \\
\hline $2-17$ & + & - & + & - & - \\
\hline $2-18$ & + & - & + & - & - \\
\hline $2-19$ & - & + & + & - & - \\
\hline $2-20$ & - & + & + & - & - \\
\hline $2-21$ & + & - & - & - & DR2 \\
\hline $2-22$ & + & - & - & - & DR2 \\
\hline $2-23$ & + & - & - & - & DR2 \\
\hline $2-24$ & + & - & - & - & DR3 \\
\hline $2-25$ & + & - & - & - & DR3 \\
\hline $2-26$ & + & - & - & - & DR3 \\
\hline $2-27$ & + & - & - & - & DR5 \\
\hline $2-28$ & + & - & - & - & DR5 \\
\hline $2-29$ & + & - & - & - & DR7 \\
\hline $2-30$ & + & - & - & - & DRw8 \\
\hline $2-31$ & + & - & - & - & Dw19 \\
\hline $2-32$ & + & - & - & - & Dw18 \\
\hline $2-33$ & + & - & - & - & DR2 \\
\hline $2-34$ & - & + & - & - & DR5 \\
\hline $2-35$ & - & + & - & - & DRw8 \\
\hline $2-36$ & - & + & - & - & DRw8 \\
\hline
\end{tabular}

characteristic of RA extending beyond the synovia (Table V). Immunogenetically, this patient subset was the most homogeneous patient group. 17 of 18 patients expressed the $B 1{ }^{*} 0401$ allele $(P=0.00001)$ (Table II). One patient carried one unusual $B 1$ gene which could be assigned as variant of the $B 1^{*} 1402$ allele by sequencing the B1 products. In $72.2 \%$ of the patients only one DR4 PCR product was amplified by the allele-specific primer sets. These 13 patients were potentially homozygous for the B1 locus or had inherited two distinct variants of the B1 allele presenting phenotypically as homozygous for the DR4. In nine of these patients, oligonucleotide probing for HVR3 specific sequences exclusively detected the product of the B $1{ }^{*} 0401$ allele, one patient expressed only the B ${ }^{*} 0404$ allele, three were $B 1 * 0401 / 0404$ heterozygous. Sequence analysis revealed that the HLA-DR oligotyping was unambiguous and that these nine patients were truly homozygotes. Homozygos-
Table V. Clinical Characteristics and HLA-DRB1 Alleles in Patients with Nodules and Major Organ Involvement

\begin{tabular}{lll}
\hline & & HLA-B1 alleles \\
\hline $3-1$ & Rheumatoid leg ulcer & $0401 / 0401$ \\
& Neuropathy & \\
$3-2$ & Rheumatoid leg ulcer & $0401 / 0401$ \\
& Felty's syndrome & \\
$3-3$ & Cutaneous vasculitis & $0401 / 0401$ \\
$3-4$ & Cutaneous vasculitis & $0401 / 0401$ \\
$3-5$ & Felty's syndrome & $0401 / 0401$ \\
$3-6$ & Rheumatoid lung disease & $0401 / 0401$ \\
$3-7$ & Rheumatoid vasculitic mononeuritis & $0401 / 0401$ \\
$3-8$ & Systemic vasculitis & $0401 / 0401$ \\
$3-9$ & Cutaneous vasculitis & $0401 / 0401$ \\
$3-10$ & Rheumatoid lung disease & $0404 / 0404$ \\
$3-11$ & Central nervous system vasculitis & $0401 / 0404$ \\
$3-12$ & Rheumatoid vasculitic mononeuritis & $0401 / 0404$ \\
$3-13$ & Felty's syndrome & $0401 / 0404$ \\
& Rheumatoid ulcer & \\
$3-14$ & Felty's syndrome & $0401 / D R 2$ \\
$3-15$ & Cutaneous vasculitis & $0401 / D R 2$ \\
& Felty's syndome & \\
& Mucosal vasculitis & $0401 /$ DR3 \\
$3-16$ & Cutaneous vasculitis & \\
& Neuropathy & $0401 /$ Dw19 \\
$3-17$ & Cutaneous vasculitis & $1401 / 1402$ \\
$3-18$ & Cutaneous vasculitis & \\
& Neuropathy & \\
& Pulmonary disease & \\
& &
\end{tabular}

ity for the $\mathrm{B} 1 * 0401$ and the $\mathrm{B} 1 * 0404$ allele, respectively, was a rare event in the normal controls, we have not seen homozygous individuals for any of these variants among 47 control individuals. The predominance of the DRB $1 * 0401 / 0401$ homozygotes was unexpected and clearly distinguished the patients with extraarticular disease from the nonnodular as well as the nodular patients (Table III, $P=0.0003$ ). The $\mathrm{B}^{*} 0101$ allele was not identified at all among the patients with the most aggressive form of the disease $(P=0.0086)$. Three patients carried the B $1 * 0401$ allele together with $B 1 * 0404 / 8$, a combination which was also frequently encountered among nodular patients. The minority of patients had only one disease-associated $B 1$ gene, which in all cases was unequivocally identified as $B 1^{*} 0401$. The second nonassociated haplotypes coexpressed by these patients included DR2, DR3, and Dw19. Thus, as already seen for nodular RA patients, the patient population with life-threatening manifestations of RA frequently expressed a combination of two disease-associated haplotypes $(P$ $=0.0001$ compared to patients with nonnodular disease). In contrast to nodular patients the genotype was characterized by a strong distortion of the $\mathrm{B} 1{ }^{*} 0401$ frequency, especially for the homozygous state of that gene $(P=0.0003)$.

\section{Discussion}

In this study, we have investigated the hypothesis that the disease-associated molecular polymorphism of HLA class II genes 
not only represents a susceptibility gene to develop RA, but also affects the expression of the disease in distinct patient subsets. Our data demonstrate that the B ${ }^{*} 0401$ allele of the HLADRB1 gene supports progression of RA to extraarticular manifestations and that homozygosity of the $B 1 * 0401$ allele is tightly linked with the most severe presentation of the disease. Understanding the codominant function of the disease-associated $\mathrm{B} 1$ genes in disease expression might allow prospective definition of patients at risk of developing potentially fatal manifestations of RA and provide clues to the pathomechanisms distinguishing patients with mild and severe disease.

We have studied the molecular polymorphism of the HLADRB1 gene by PCR amplification and allele-specific oligonucleotide probing in three patient subsets representing the spectrum of RA from the milder form of erosive RA to the lifethreatening complications of major organ involvement. Stringent criteria were used to assign patients to the different subsets. To prevent misassignment of a patient due to limited disease duration and unequal follow-up, we have not enrolled any patient with nonerosive disease and have matched the patient population for age and disease duration. The high frequency of the HLA-DR4 haplotype in all three disease categories strongly supports the association of RA with that haplotype. Previous studies have reported that $65-80 \%$ of RA patients carry the disease-associated haplotype (20). It has been hypothesized that the incompleteness of the linkage can be explained by the presence of a particular nucleotide sequence in HLA-DR4- patients, which is shared by the HLA-DRB1 alleles *0404, *0405, *0101, and *1402 and has only one conservative substitution in DRB1*0401. The strong association in our study with HLA-DR4 is most likely due to the patient selection which required bony erosions as one entry criteria. Whereas the patient group with the mildest disease manifestation, group 1, had two individuals not expressing the diseaseassociated genetic polymorphism and five patients solely expressing the HLA-DRB1*0101 or ${ }^{*} 1402$ gene, patients with nodular and extraarticular disease were exclusively positive for the disease-associated HLA-DRB1 product and, with one exception, HLA-DR4 ${ }^{+}$. These data suggest a hierarchy of susceptibility with a more pronounced association with HLADRB $1 * 0401$ or B $1^{*} 0404$ in RA patients with severe disease $(21,22)$.

The chief finding in our study is the high prevalence of B1*0401 homozygous patients in the group with the most severe disease. To rule out the possibility that we had missed an HLA-DRB1 gene in the initial PCR amplification, we chose to sequence all HLA-DRB1 locus products in individuals categorized as homozygous. This approach confirmed that these patients only had a single B1 locus product. Homozygosity for the HLA-DRB1*0401 allele is not a frequent event in a normal population. We have not seen any DRB $1^{*} 0401$ homozygous individuals in a group of $\mathbf{4 7}$ healthy donors. Thus, homozygosity of the DRB 1*0401 allelic variant might potentially be used as a prognostic marker in patients diagnosed with RA. A codominant function of HLA-DRB1 alleles has been reported for children with seropositive juvenile arthritis who had a higher than expected frequency of carrying both the $B 1 * 0401$ and B1*0404 alleles (23). These observations suggest that such a double dose of susceptibility genes may predispose to an earlier onset of the disease and to more severe disease manifestations.

Although patients with extraarticular manifestations in this study did not comprise a clinically homogeneous subset they were genetically surprisingly homogeneous (Table V). There was no evidence that disease manifestations at certain organ systems translated into immunogenetic differences. Progression to severe disease, including rheumatoid lung disease, rheumatoid vasculitis, Felty's syndrome, and rheumatoid vasculitic mononeuritis, was highly associated with the codominant expression of two * 0401 alleles and, to a much lesser extent, of the B1*0401 and B1*0404/8 alleles (Table III). Thus, a model emerges in which a particular molecular polymorphism, when present as a homozygous genotype, determines a wide variety of pathological processes suggesting a common denominator amongst all the different organ manifestations of RA.

The important contribution of the second haplotype to disease expression was also seen for nodular patients without major organ involvement. In contrast to the most severely affected patients, homozygosity for the B $1^{*} 0401$ subtype of the HLADR4 haplotype was infrequent. However, the majority of patients combined two distinct haplotypes carrying the RA-associated motif within the HVR3 region of the HLA-DRB1 first domain. The increased frequency of HLA-DR1/DR4 and HLA-Dw4/Dw14 heterozygous patients might reflect a gene dose effect. We have not seen a nodular patient who typed HLA-DR4 negative. It is therefore conceivable that $\mathrm{B} 1{ }^{*} 0401$ alone or $\mathrm{B} 1^{*} 1402$ alone may not play an independent role in patients with nodular disease. This result challenges the hypothesis that the disease susceptibility for RA can be conferred by a short stretch of amino acids encoded by the HVR3 of the HLA-DRB 1 chain. Obviously, the framework of the $\beta 1$-chain into which the RA-associated region has been integrated plays a major role in determining the contribution of the key structural element encoded by the susceptibility sequence stretch.

Evidence for a critical contribution of haplotype combinations has been provided for other HLA-associated diseases. An increased risk for the heterozygous genotype has been demonstrated for diabetes mellitus, celiac disease, and primary Sjögren's syndrome (24-26). The absence of a protective HLADR or DQ allele or novel transencoded DQ molecules have been discussed as putative mechanisms. A major influence of certain DR-DQ combinations strongly affecting disease susceptibility as a pair has also been reported for diabetes mellitus (27). These models do not represent possible or likely explanations for the increased frequency of HLA-DRB 1*0401 homozygosity in RA patients with major organ involvement. It is highly unlikely that all DRB1 alleles except DRB1*0401 express the same protective ability and thus homozygosity for HLA-DRB $1 * 0401$ is required for the full expression of the disease.

It has been suggested that the disease-associated sequence stretch which is homologous amongst the four different disease-associated haplotypes functions by binding selected peptide fragments and presenting them to the TCR. In this model, the cell surface expression of the HLA-DR molecule expressing the disease-associated motif in the homozygous host would be double the amount present in a heterozygous patient. Increased expression of a particular HLA-DR molecule could increase the amount of antigenic peptide presented on the surface of an antigen presenting cell. Studies in homozygous and heterozygous mice do not support the hypothesis that such an increase would be of functional significance and would have a major effect on $\mathrm{T}$ cell responses. In addition, it is difficult to 
conceive how the disease-modifying influence of the DRB $1{ }^{*} 0401$ homozygosity on RA expression and in particular on the extraarticular manifestations could relate to the antigen presenting function of an HLA-DR molecule presenting an arthritogenic peptide.

In addition to their antigen-binding properties, HLA molecules play a critical role in shaping the T cell repertoire. Based upon the recent crystallographic resolution of an HLA molecule, the HVR3 encoded sequence predisposing to RA is predicted to encode for the $\alpha$-helical wall surrounding the antigenbinding groove (28). Whether this region of the molecule is more important in binding the antigen peptide in the groove or interacting with the TCR is presently unresolved. In previous studies, we have shown that the sequence polymorphism encoded by the HVR 3 is instrumental in determining $T$ cell responses $(29,30)$. The repertoire of recruited $T$ cell specificities interacting with the disease-associated epitopes is limited in that two TCR $\mathrm{V} \beta$ families are preferentially selected (31). These data suggested that the disease-associated epitope is functional in directly interacting with the TCR and thus may be involved in the formation of the T cell repertoire. Provided that the $\mathrm{T}$ cell repertoire of a responder is shaped by the available MHC molecules, the presence of a single or two different HLA-DRB 1 genes would be of utmost importance. The homozygous genotype would be missing the contribution of a second haplotype. Progression to advanced and aggressive disease might be due to the function of $T$ cell specificities which are either preferentially selected by the DRB $1^{*} 0401$ allele or the lack of protective $T$ cell specificities which would be selected by a DRB1 non-*0401 allele. A role of the DRB1*0401 homozygous state in $T$ cell selection would best explain the influence of the DRB1*0401 gene product which extends clearly over and above the dominant effect of DRB1*0401 in susceptibility to RA.

\section{Acknowledgments}

We are indebted to our clinical colleagues Drs. D. Conn, W. Ginsburg, G. Hunder, H. Luthra, E. Matteson, C. Michet, and D. O'Duffy for contributing patients; to Dr. W. O'Fallon for statistical consultation; to K. Hicok and T. Reed for technical assistance; to Toni Buss for excellent assistance in the preparation of the manuscript; and to the patients who participated in the study.

Supported by grants 76 and 77 from the Minnesota Arthritis Foundation, AI31 155-01 B and AI31 155-01C from the National Institutes of Health and the Mayo Foundation. Dr. C. M. Weyand is the recipient of an Arthritis Investigator Award from the Arthritis Foundation.

\section{References}

1. Harris, E. D., Jr. 1989. The clinical features of rheumatoid arthritis. In Textbook of Rheumatology. W. N. Kelley, E. D. Harris, S. Ruddy, and C. B. Sledge, editors. W. B. Saunders Co., Philadelphia. 943-980.

2. Harris, E. D., Jr. 1990. Rheumatoid arthritis: pathophysiology and implications for therapy. N. Engl. J. Med. 322:1277-1289.

3. Stastny, P. 1978. Association of the B-cell alloantigen DRw4 with rheumatoid arthritis. N. Engl. J. Med. 298:869-871.

4. Nepom, G. T., S. Holbeck, C. E. Seyfried, K. P. Wilske, and B. S. Nepom. 1986. Identification of HLA-Dw14 genes in DR4-positive RA. Lancet. ii:10021004.

5. Goronzy, J., C. M. Weyand, and C. G. Fathman. 1986. Shared T cell recognition sites on HLA class II molecules of patients with seropositive rheumatoid arthritis. J. Clin. Invest. 77:1042-1049.

6. Schiff, B., Y. Mizrachi, S. Orgad, M. Yaron, and I. Gazit. 1982. Association of HLA-Aw31 and HLA-DR1 with adult rheumatoid arthritis. Ann. Rheum. Dis. 41:403-406.

7. Willkens, R. F., G. T. Nepom, C. R. Marks, J. W. Nettles, and B. S. Nepom. 1991. The association of HLA-Dw16 with rheumatoid arthritis in Yakima Indians: further evidence for the "shared epitope" hypothesis. Arthritis Rheum. $34: 43-47$.

8. Weyand, C. M., and J. J. Goronzy. 1989. Seropositive rheumatoid arthritis is associated with T-cell epitopes encoded within the third hypervariable region of the HLA-DR 1 and the HLA-DR4 haplotype. In Immunobiology of HLA, Immunogenetics and Histocompatibility. B. Dupont, editor. Springer Verlag New York Inc. II:422-425.

9. Gregersen, P. K., J. Silver, and R. J. Winchester. 1987. The shared epitope hypothesis. Arthritis Rheum. 30:1205-1213.

10. Nepom, G. T., J. Hansen, and B. Nepom. 1987. The molecular basis for HLA class II associations with rheumatoid arthritis. J. Clin. Immunol. 7:1-7.

11. Arnett, F. C., S. M. Edworthy, D. A. Bloch, et al. 1988. The American Rheumatism Association 1987 revised criteria for the classification of rheumatoid arthritis. Arthritis Rheum. 31:315-324.

12. Vollertsen, R. S., and D. C. Conn. 1990. Vasculitis associated with rheumatoid arthritis. Rheum. Dis. Clin. North Am. 16:445-462.

13. Gao, X., N. J. Olsen, T. Pincus, and P. Stastny. 1990. HLA-DR alleles with naturally occurring amino acid substitutions and risk for development of rheumatoid arthritis. Arthritis Rheum. 33:939-946.

14. Weyand, C. M., K. C. Hicok, and J. J. Goronzy. 1991. Nonrandom selection of $T$ cell specificities in anti-HLA-DR responses: sequence motifs of the responder HLA-DR allele influence T cell recruitment. J. Immunol. 147:70-78.

15. Bodmer, J. G., E. Albert, W. F. Bodmer, B. Dupont, B. Mach, W. R. Mayr, T. Sasazuki, G. M. Th Schreuder, J. L. Strominger, A. Svejgaard, et al. 1990. Nomenclature for factors of the HLA system. Immunogenetics. 31:131140.

16. Sarkar, G., and S. S. Sommer. 1989. Access to a messenger RNA sequence or its protein product is not limited by tissue or species specificity. Science (Wash. DC). 244:331-334.

17. Stoflet, E. S., D. D. Koeberl, G. Sarkar, and S. S. Sommer. 1988. Genomic amplification with transcript sequencing. Science (Wash. DC). 239:491-494.

18. Gregersen, P., M. Shen, Q. Song, P. Merryman, S. Degar, T. Seki, J. Maccari, D. Goldberg, H. Murphy, J. Schwenzer, et al. 1986. Molecular diversity of HLA-DR4 haplotypes. Proc. Natl. Acad. Sci. USA. 83:2642-2646.

19. Petersdorf, E. W., A. G. Smith, E. M. Mickelson, P. J. Martin, and J. A. Hansen. 1991. Ten HLA-DR4 alleles defined by sequence polymorphisms within the DRB1 first domain. Immunogenetics. 33:267-275.

20. Tiwari, J., and P. Terasaki. 1985. HLA and disease associations. Springe Verlag New York Inc.

21. Westedt, M. L., F. C. Breedveld, G. M. T. Schreuder, J. D'Amaro, A. Cats, and R. R. P. De Vries. 1986. Immunogenetic heterogeneity of rheumatoid arthritis. Ann. Rheum Dis. 45:534-538.

22. Young A. D. Jaraquemada, J Awad et al 1984. Association of HLADR4/Dw4 and DR2/Dw2 with radiologic changes in a prospective study of patients with rheumatoid arthritis. Preferential relationship with HLA-Dw rather than HLA-DR specificities. Arthritis Rheum. 27:20-25.

23. Nepom, B. S., G. T. Nepom, E. Mickelson, J. G. Schaller, P. Antonelli, and J. A. Hansen. 1984. Specific HLA-DR4-associated histocompatibility molecules characterize patients with seropositive juvenile rheumatoid arthritis. J. Clin. Invest. 74:287-291.

24. Svejgaard, A., and L. P. Ryder. 1981. HLA genotype distribution and genetic models of insulin-dependent diabetes mellitus. Ann. Hum. Genet. 45:293-298.

25. Sollid, L. M., G. Markussen, J. Ek, H. Gjerde, F. Vartdal, and E. Thorsby. 1989. Evidence for a primary association of celiac disease to a particular HLADQ $\alpha / \beta$ heterodimer. J. Exp. Med. 169:345-350.

26. Harley, J. B., M. Reichlin, F. C. Arnett, E. L. Alexander, W. B. Bias, and T. T. Provost. 1986. Gene interaction at HLA-DQ enhances autoantibody production in primary Sjögrens syndrome. Science (Wash. DC). 232:1145-1147.

27. Sheehy, M., Jr., S. J. Scharf, J. R. Rowe, M. H. Nemede Gimenez, L. M. Meske, H. A. Erlich, and B. S. Nepom. 1989. A diabetes-susceptible HLA haplotype is best defined by a combination of HLA-DR and -DQ alleles. J. Clin. Invest. 83:830-835.

28. Brown, J. H. T. Jardetzky, M. A. Saper, B. Samraoui, P. J. Bjorkman, and D. C. Wiley. 1988. A hypothetical model of the foreign antigen binding site of class II histocompatibility molecules. Nature (Lond.). 332:845-850.

29. Weyand, C. M., and J. J. Goronzy. 1989. Mapping of allospecific T-cell recognition sites encoded by the HLA-DR4 $\beta_{1}$-chain. Hum. Immunol. 24:133143.

30. Weyand, C. M., and J. J. Goronzy. 1990. Disease-associated HLA determinants in patients with seropositive rheumatoid arthritis: functional role in antigen-specific and allogeneic T cell recognition. J. Clin. Invest. 85:1051-1057.

31. Weyand, C. M., U. Oppitz, K. C. Hicok, and J. J. Goronzy. 1992. Selection of $\mathrm{T}$ cell receptor $\mathrm{V} \beta$ elements by HLA-DR determinants predisposing to rheumatoid arthritis. Arthritis Rheum. In press. 